\title{
Context-Aware Middleware Support for the Nomadic Mobile Services on Multi-homed Handheld Mobile Devices
}

\author{
Pravin Pawar ${ }^{1}$, Bert-Jan van Beijnum ${ }^{2}$, Arjan Peddemors ${ }^{3}$, Aart van Halteren ${ }^{4}$ \\ ${ }^{1,2}$ Architecture and Services for Networked Applications Group \\ Department of Computer Science \\ University of Twente, The Netherlands. \\ \{P.Pawar,Beijnum\}@utwente.nl \\ ${ }^{3}$ INCA Group, Telematica Instituut, The Netherlands. \\ Arjan.Peddemors@telin.nl \\ ${ }^{4}$ Philips Research, Eindhoven, The Netherlands. \\ Aart.van.Halteren@philips.com
}

\begin{abstract}
Nowadays, a variety of handheld mobile devices are capable of connecting to the Internet using multiple network interfaces. This is referred to as multi-homing. In addition to this, enriched computation resources allow them to host nomadic mobile services and provide these services to the clients located anywhere in the Internet. Potential advantages of multi-homing for nomadic mobile services typically includes: an increased service availability and improved service performance. However, applications running on the handheld mobile devices either do not, or cannot, exploit multi-homing.

In this paper we address the problem of providing infrastructural support to the nomadic mobile services that can fully exploit multi-homing. To this end we propose to incorporate multi-homing functionality and support in a middleware layer to reduce the complexity of the design and maintenance of these services. The proposed solution uses a context-aware computing approach to realize this functionality. We report the initial experimental results in the remote telemonitoring of a patient equipped with a Body Area Network.
\end{abstract}

\section{Introduction}

Today, mobile devices are often equipped with multiple network interfaces such as Wi-Fi, GSM, UMTS and USB, which enable them to connect to the Internet. Multi-homing is the capability of a mobile device to connect to multiple IP networks [1]. Multihoming is utilized in several approaches (e.g. [1], [2], $[3],[4],[5],[6])$ to provide the mobile devices with high availability Internet connectivity, redundancy, fault tolerance, load balancing, cost-based communication decisions, low latency handoff and QoS improvement. However, these approaches consider mobile devices such as laptops and mobile routers. Although multiple Internet access technologies are supported and in principle multiple Internet access providers are available, many applications designed and running on the handheld devices such as mobile phones and PDAs do not exploit this multi-homing to the maximum possible extent or worse, do not consider it at all. One of the reasons for this lack of support is that the operating system of a mobile device exposes functionality which is often technology and vendor specific; which makes it difficult to obtain the state of the currently available network resources.

Service Oriented Architecture (SOA) paradigm allows flexible service offering and run-time service selection on the Internet. Traditionally, mobile devices take on a service consumer role. However, today these devices have the resource requirements to host services, and can become the part of the service discovery network. For instance, in [7] a proxy based middleware is presented for the development and deployment of application services hosted on a mobile device. In [8] and [9] a lightweight infrastructure is proposed to host web services on a mobile device. We name such a service as Nomadic Mobile Service (NMS). A mobile device in the role of service provider enables, amongst others, entirely new scenarios and end-user services. For example, in the Mobi-Health domain, a remote patient tele-monitoring service collects the vital signs of the patient wearing a Body Area Network (BAN) and make this data available on demand to the health-care professionals, who may be monitoring the patient in real-time [10]. 
There are two notable characteristics of a NMS which are: 1) The role of a mobile device changes from the consumer to producer and hence the available uplink bandwidth is critical; 2) The service offering is influenced by the characteristics of the communication environment of a mobile device since the service roams with the device it is hosted on.

Considering these aspects, it is important to provide multi-homing support to the NMSes which will enable: 1) Selection of the communication network which satisfies their bandwidth requirements and thus provides data transfer resiliency; 2) Handover from one network to another if the connectivity is no longer available and thus maintain network transparency; 3) Preservation of the current data transfer sessions during the vertical handover and thus improve the client experience.

Further, incorporating this functionality in the middleware will reduce the complexity involved in the development and maintenance of the nomadic mobile services and will promote their reuse.

We take a context-aware computing approach, following the principles proposed by Henricksen et al. [11] to provide the multi-homing support. The features of our work in progress include: 1) Modular architecture consisting of a communication context source, context processor and context reasoner components interfacing with the NMS provisioning middleware proposed in [7]; 2) Use of multi-homed handheld mobile devices; 3) Validation in the healthcare domain for the real-time tele-monitoring of a patient equipped with the Body Area Network.

This paper is organized as follows. Section 2 of this paper briefly describes our middleware platform Mobile Service Platform (MSP). We further elaborate on the requirements for multi-homing NMSes. Section 3 , presents a context-aware middleware architecture and prototype implementation. In Section 4 shows an experimental set-up using a remote patient telemonitoring service and reports the initial validation results. Section 5 discusses the related work. Section 6 concludes the paper and discusses the future research.

\section{Requirements for Multi-Homing Nomadic Mobile Services}

Mobile Service Platform (MSP) is a middleware which facilitates the design, development and deployment of nomadic mobile services. In the sequel we present an overview of MSP. In order to enable multi-homing NMS, the requirements are to be understood and articulated, this is done subsequently.

\subsection{Mobile Service Platform}

A nomadic mobile service designed using MSP consists of two components: 1) An application realizing a service running on the mobile device (referred to as a device service); and 2) a representation of the device service in the fixed network which is referred to as a surrogate. The surrogate functions as a proxy for the device service and participates in the service discovery network. A surrogate host is responsible for the management of surrogates. MSP consists of three main parts: 1) $M S P-I O$ resides on a mobile device and interacts with the device service. 2 ) $M S P$-Interconnect resides at the surrogate host and interacts with the surrogate. 3) MSP-Messages specifies the structure of messages exchanged between the device service and the surrogate. MSP uses HTTP as a data transfer protocol.

MSP support three interactions between the device service and surrogate as follows:

- One-Way messaging: The One-Way messaging allows for unacknowledged message delivery between the device service and its surrogate. This kind of message does not have a corresponding reply.

- Request-Response messaging: The RequestResponse messaging supports reliable message delivery. The request message must have a corresponding reply message.

- Streaming interaction: Streaming supports exchange of continuous data (streams) from the device service to the surrogate.

MSP middleware uses dedicated control plane interactions for control, monitor and lifecycle management of the NMS. An example of this message is the Keep-Alive message, which is sent by the device service at fixed intervals and the surrogate host acknowledges this message by sending a response.

\subsection{Multi-homing Requirements of the Nomadic Mobile Services}

Providing multi-homing support can be of vital interest to the NMS. To illustrate this, consider the application of NMS for tele-monitoring of a patient. Assume a patient is equipped with a Body Area Network, by means of which a set of vital signs is monitored (e.g. ECG, Oxygen Saturation level, Heart Rate). A medical specialist needs, for instance for the purpose of a treatment session, to monitor these vital signs in real-time. Medical protocols and practices prescribe strict requirements on the quality of the vitals sign data set, such as sampling rate, sample size. 
Depending on the type of vital signs to be monitored, for realistic scenario's application data streams from 28 kbps $-40 \mathrm{kbps}$ typically occur. However, in practice, this amount of bandwidth may not be provided by the communication network a patient is connected to (e.g. GPRS). Hence it is desired that whenever a high capacity network (e.g. WLAN) is available, it should be preferred over the low capacity network (e.g. GPRS). Providing multi-homing support to NMS will facilitate such behavior. Due to the inversion of the role from consumer to producer and considering the role played by the nomadic mobile services, the multihoming support to NMS should focus on the following aspects:

Optimal Network Selection: The existing cellular networks are characterized by the lower uplink bandwidth and higher downlink bandwidth. (e.g. GPRS has uplink bandwidth of $20 \mathrm{kbps}$ as compared to $40 \mathrm{kbps}$ downlink.) In practice, the bandwidth available to the application is usually less than the theoretical bandwidth [12]. The bandwidth experienced by a mobile device is also affected by the number of devices sharing the wireless link [13]. MSP must support network handover based on the available uplink bandwidth and application bandwidth requirements.

Low Latency: Considering the critical situations in which the NMSes are employed, low latency is desired from the selected network. E.g. In the tele-monitoring case, the caregiver or paramedics need to be informed about the critical condition of a patient within the time to react. The latency of the available wireless networks varies widely. For the streaming data, the latency during the vertical handoff is also critical.

Session Handling: In addition to the reasoning support, during the network handover, the MSP should be able to preserve the ongoing data transfer session. Moreover, it is likely that the new network has less bandwidth than the old network. In this case, there should be mechanisms to buffer the data temporarily, monitor the progress of data transfer and instruct the services to reduce the data transfer rate.

Economic Internet Availability: Provided that the mobile user roams in a variety of networks and the cost of connectivity varies depending on the ISP, it is desired to select a network which provides economic Internet connectivity. However, in some cases, such as when a patient suffers from epileptic seizure, cost may not be of the concern but availability is the most important.

Reasoning Support: To enable multi-homing for the NMSes, the MSP should be aware of the capabilities of a mobile device in terms of the supported and available network interfaces, their properties as well as should be able to use the appropriate network. A reasoning support at the middleware could possibly make use of the following factors to determine the suitability of the network:

i) Theoretical QoS characteristics such as bandwidth, delay and jitter of the available network interfaces; ii) Actual throughput offered by various networks; iii) Monetary cost to use the network; iv) Energy requirements to use the available interfaces; v) Criticality of the nomadic mobile service.

Additionally, it is also desired to get some feedback from the NMS to reflect on this reasoning support.

Context Sensing and Processing: The context sources should provide real-time and accurate context information as well as should notify changes of context (e.g. availability of another network). In this case, the context information consists of the (see the text on reasoning support above) factors which contribute to the reasoning support.

In the current version of the architecture and implementation, we fulfill the requirements related to network handover and context-awareness. We include a limited reasoning support as well. Pl. note that our current design is further scoped by the context sources that we consider and to which MSP interfaces. We are researching various architectural and implementation aspects to handle other requirements.

\section{Architecture and Implementation of Context-Aware Mobile Service Platform}

We consider communication context of a mobile device that consists of three different elements: network context in the form of cross-layer information, the state of the (operating) system facilities relevant to the communication with other endpoints, and the state of facilities available in the network infrastructure that support this communication.

\subsection{Architectural Design}

Figure 1 shows the high-level architecture of Context-Aware MSP. The following components contribute to this architecture:

- Communication and computation hardware: The handheld mobile devices considered here are equipped with multiple network interfaces to allow for the concurrent access to multiple networks.

- Device Operating System: Most modern operating systems for high-end mobile devices provide various means to observe and control the network interfaces available on the device. Additionally, 
the OS may inform applications on the state of IP settings and routes associated with currently active networks and provide means to establish transport layer connections through the standardized Socket API. Information on link and network layer state, however, is provided through different APIs often technology and vendor specific - where it is left to the developer to connect the entities at link, network and transport layer.

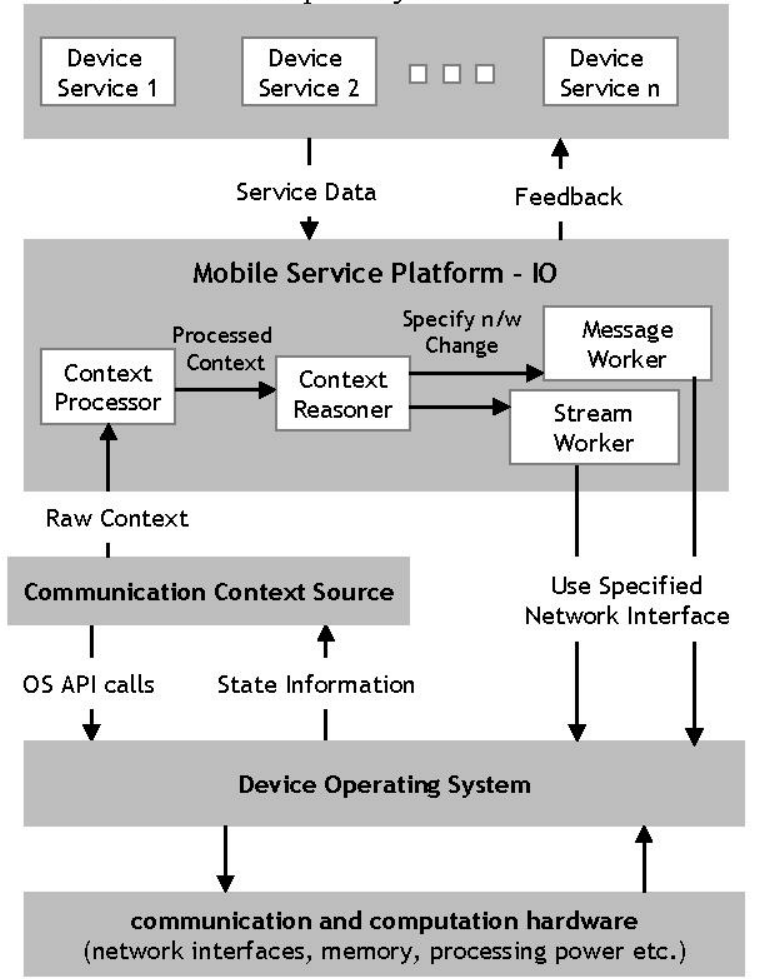

Fig. 1: High Level Architecture of ContextAware MSP

- Communication Context Source (CCS): To obtain a consistent cross-layer view of the available network resources, the MSP uses the Communication Context Source (CCS). CCS provides the MSP with the right level of information to make decisions on the usage of the available networks. This information is structured according to the Network Resource Model (NRM) introduced in [14], represented using XML, and allows the MSP to retrieve information about the protocol stack. The NRM defines in an explicit manner the relationship between the available entities at the link, network and transport layers, showing the application the relationship between, for example, the available outgoing IP paths (routes) and the existing links over which these paths are realized. The CCS offers a subscription mechanism to send messages about network changes.

- Context Processor: On activation, the context processor obtains the initial network state from the CCS and subscribes to it for the context change events. Such an event is triggered when a mobile device joins a new network or disconnects from one of the connected networks. After receiving every context change event, context processor updates the current network state and sends this information to the Context Reasoner.

- Context Reasoner: The context reasoner parses the XML representation of the current network state to extract information about the network interfaces that provide internet connectivity, their availability, bandwidth, MTU size and name. The current criteria for selecting the network consider only the network bandwidth and availability. If the selected network interface is different than the one currently in use, the context reasoner informs the Message Worker and Stream Worker components (see below) about the availability of a new network interface. In the case of unavailability of any network interface, the device service is notified of this.

- Message Worker: MSP-IO consists of a Message Worker component to receive one-way and request-reply messages from the device service and send it to its surrogate. The Message Worker is also responsible for handling the incoming messages from the surrogate. MSP-IO additionally uses Message Worker to send control messages to the surrogate host. Internally, a Message Worker uses the network interface specified by the Context Reasoner to post the message data using HTTP.

- Stream Worker: The MSP-IO Stream Worker provides a buffer to the device service to which a service constantly writes the streaming data. Stream worker opens a connection to the surrogate of a device service via the network interface specified by the Context Reasoner, reads data from the buffer and transmits it to the surrogate host.

\subsection{Implementation}

The CCS implementation is based on the Network Abstraction Layer (NAL) reference implementation for Windows CE [14] with the extensions to generate network resource descriptions in XML. As most of the other components are implemented in Java, and the NAL is in native code, a client DLL was developed to 


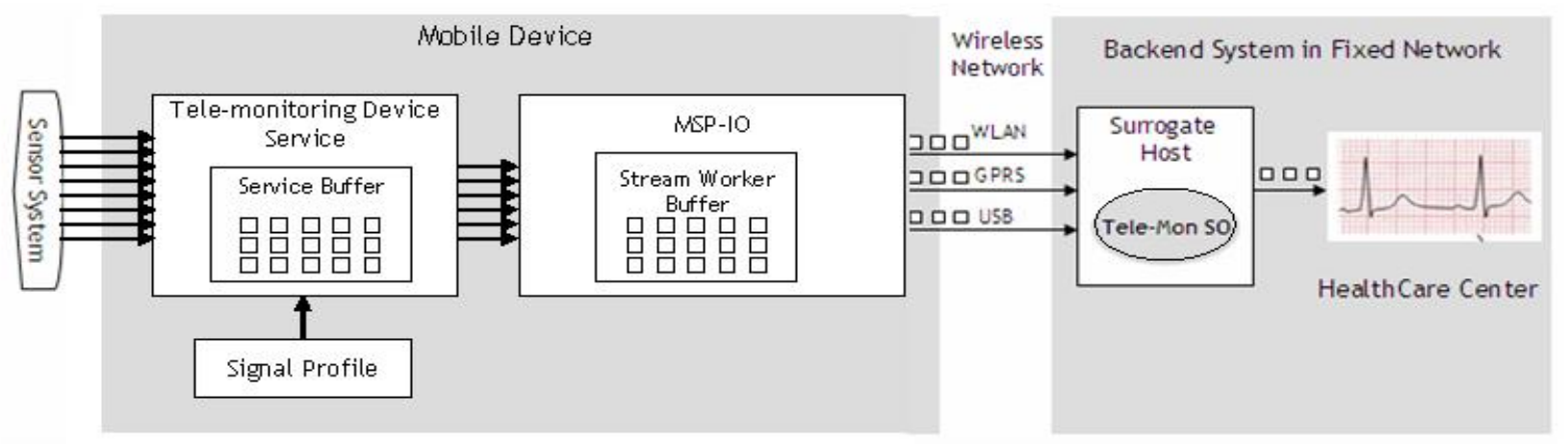

Figure 2: Overview of the system to validate context-aware MSP for the tele-monitoring service

interface the NAL with the JVM All communication betw een the NAL and Context Processor, including the initiation of the subscription, is realized using XML. The client DLL maintains a full-duplex pipe to NAL to send XML back and forth.

MSP design is based on Jini technology [15]. The communication between the Device Service and Surrogate is specified by Jini Surrogate Architecture Specification [16]. We have developed an HTTP implementation (referred to as HTTPInterconnect) of the Interconnect protocol specified in [16] so that the device service is able to communicate with its surrogate. The device service is usually implemented using J2ME technology. For more information on MSP, we refer to [7].

Context Processor, Context Reasoner, Message Worker and Stream Worker are a part of MSP-IO package (refer to Figure 1). Context Processor is a thread which interfaces with the CCS using Java Native Interface (JNI). The Context Reasoner uses KXML library [17] to parse the XML representation of the network state. The Message Worker and Stream Worker are also threads and use Apache HTTPClient library [18] to send messages and transmit stream to the surrogate host. The Context Reasoner converts the IP address of the best network interface to the InetAddress and changes the hostConfiguration which is later used by the HTTPClient to open an HTTP connection. In the case of unavailability of the Intemet connection, a device service is notified by means of a Java exception.

\section{Validation}

For the healthcare industry, mobile applications prov ide an opportunity to offer better care and serv ices to the patients and a more flexible and mobile way of communicating with the patients and caregivers [19]. Figure 3 shows the application of nomadic mobile serv ices for remote tele-monitoring in the health-care domain. The tele-monitoring service is integrated with the context-aware MSP for validation into the integrated demonstrator of AWARENESS project [20].

The tele-monitoring service monitors a patient's vital signals through a BAN. A BAN consists of sensor systems, actuator systems, communication and processing facilities [21]. The current BAN implementation employs a Qtek9090 PDA, which runs Windows Mobile 2003 and a J2ME compliant JVM, as the Mobile Base Unit (MBU). MSP transmits the BAN vital signs received by the tele-monitoring device service to the tele-monitoring surrogate which later sends them to the Jini client located at the health-care center. Figure 4 shows the ov erview of our system to validate context-aware MSP for the tele-monitoring of a patient. The description of various components is as follows:

- Sensor system: A BAN sensor system processes vital signs measured by sensors attached to the patient's body, and outputs multiple channels of patient vital signs data. It communicates with the mobile dev ice using Bluetooth.

- Signal Profile: The signal profile informs the telemonitoring device service about the signals to be sent to the health-care professions. This profile varies as per the kind of treatment patient is receiving. Herewith we use two signal profiles one for the Cardio-Vascular diseases and the other for Generic tele-monitoring. These profiles are calibrated in the Health-Services 24 project [22]. The bandwidth requirements including the $10 \%$ transmission protocol ov erheads [23] are 28 $\mathrm{kbps}$ and $40 \mathrm{kbps}$ for the cardio-vascular profile and generic tele-monitoring profile respectively. For the description of the data generated by each sensor we refer to [24].

- Tele-monitoring Device Service: The telemonitoring device service receives the vital signs data over all the channels. This buffer stores the 
vital sign data up-to a predefined number of seconds (configured to approx. 60 seconds for the validation) until they are transmitted by MSP-IO.

- MSP-IO: The MSP-IO delivers the vital sign data packets to the BackEnd System depending on the available transmission capacity of the wireless communication service. The Stream Worker component also maintains an internal buffer, which stores the packets if they cannot be sent immediately over the wireless communication service. Selection of a communication network using the multi-homing mechanism influences the available capacity of the Stream Worker buffer and the tele-monitoring service buffer.

Figure 3 to Figure 5 show the results of experimental validation for the cardio-vascular profile. Similar behavior is observed for the generic tele-monitoring profile. The status bar on the top of these figures show the network selected by the context-aware MSP for the transmission of vital signs. The mobile device is connected to the GPRS network all the time. We switch on the Wi-Fi (and USB) interface and switch it off in the shown interval. The context-aware MSP automatically selects the Wi-Fi (and USB) interface because it provides higher theoretical bandwidth.

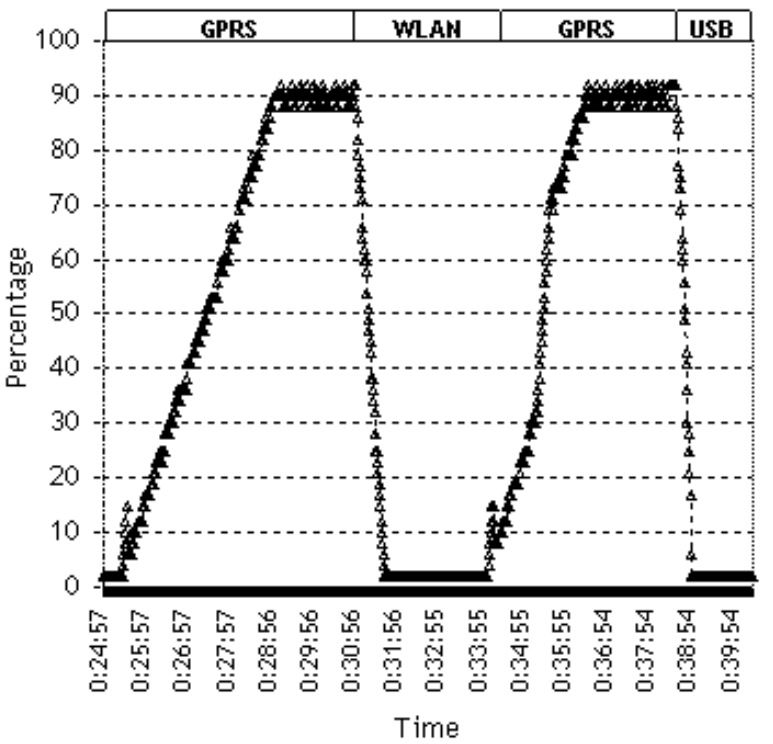

Figure 3. Tele-monitoring device service buffer fill percentage vs. time for the Cardio Signal Profile (logged on the mobile device)

Figure 3 shows the tele-monitoring service buffer fill level measured at the mobile device vs. time. It is observed that since the transmission requirements for the vital signs are higher than the bandwidth provided by the GPRS network, the service buffer fill level increases continuously; while on connecting to the WiFi and USB network interface, this level drops rapidly because of the higher bandwidth provided by these networks.

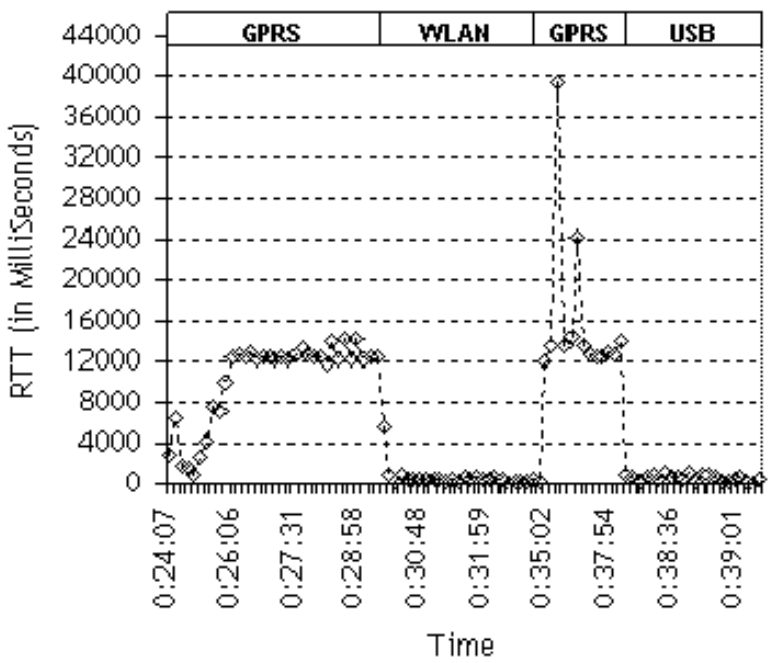

Figure 4: Keep-Alive messages RTT vs. time for the Cardio Signal Profile (logged at the surrogate)

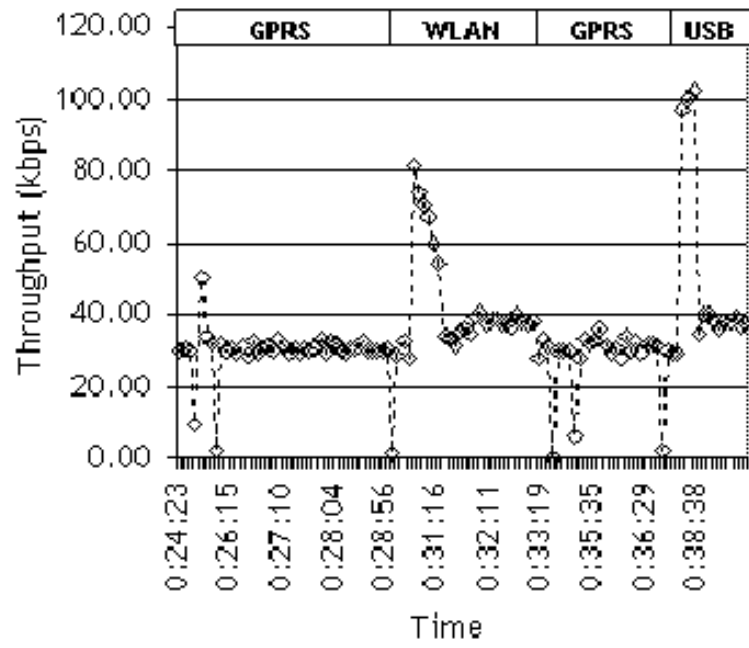

Figure 5. Vital Sign data transfer throughput vs. time for the Cardio Signal Profile (logged at the surrogate)

Figure 4 shows the Round-Trip Time (RTT) in milliseconds for the keep-alive messages logged at the surrogate vs. time. It can be observed that the Keepalive RTT is significantly higher when the mobile device is connected to the GPRS network while it is minimal for the Wi-Fi and USB networks. Figure 5 shows the amount of vital sign data received at the surrogate (in kbps) vs. time. It can be observed that the throughput provided by the GPRS connection is lower than that of Wi-Fi and USB connection. The throughput peaks occur during the change from GPRS to Wi-Fi and GPRS to USB connection. This is attributed to the emptying of the service buffer when 
the network with higher bandwidth is available. The results obtained show that incorporating multi-homing for the patient tele-monitoring service results in the flexible transmission of the vital signs of the patient.

\section{Related Work}

Most of the work related to multi-homing has been reported for mobile devices such as laptops and mobile routers supporting mobile IP. [1] exploits multihoming for low latency handoff in heterogeneous networks. It shows that multi-homing reduces handoff latency and corresponding packet loss. The experiments are simulated using NS2 for handoff between Ethernet and WLAN. The work reported in [2] describes multiple network interfaces support by using policy-based routing for the mobile IPv6 multihomed mobile nodes. It shows some quantitative results of performance evaluation using laptops. [3] proposes the in-vehicle router system to support network mobility to ensure internet connectivity and network transparency to a group of nodes connected to a mobile router in moving vehicle. The implementation environment consists of mobile IPv6 support, PDC, PHS and Wi-Fi links. [4] also proposes to use policy based handoff mechanism for mobile IPv6 enabled multi-homed hosts. [4] provides a detailed study of security risks. [5] considers the problem of providing multi-homing support in nested mobile networking. In the nested mobile networks, the hierarchy of mobile routers increases the complexity of the router selection. [6] proposes policy based hybrid approach for Ipv6 multi-homing. The hybrid approach merges both host based and router-based solutions to provide fault tolerance and load balancing capabilities. An experimental performance evaluation is conducted to evaluate the effect of various policies.

We distinguish our work from the related work described herewith in three aspects. Firstly, we employ a context-aware computing approach for multi-homing which allows clear separation of concerns for individual components and thus adds to the modular architecture. Secondly, we exploit multi-homing support for the handheld mobile devices. Thirdly, the experimental validation is conducted for the real-time remote tele-monitoring of the patient in the health-care domain.

Cross-layer information exchange (from lowerlayers to the application layer) as provided by the CCS has been investigated from different perspectives. The API presented in [25] provides an abstraction for link layer information to higher layers. It does not, however, provide a full cross-layer snapshot to applications. Additionally, the standardization efforts in the IETF Detecting Network Attachment (DNA) workgroup [26] as well as the IEEE 802.21 working group [27] also focus more on link layer abstractions.

\section{Summary and Future Work}

Multi-homing is the capability of a mobile device to connect to the multiple IP networks. This paper presents our ongoing work to exploit the multi-homing support for the services hosted on the mobile device the so called nomadic mobile services using the context-aware computing approach. This work consists of a modular architecture which includes a communication context source, context collector and context reasoner components interfacing with the Mobile Service Platform middleware. We exploit multi-homing support for the resource constrained and handheld mobile devices. The results obtained from the experimental validation in the healthcare domain for the real-time tele-monitoring of a patient confirm the benefits and feasibility of providing multi-homing support for the applications hosted on the mobile device.

In the future, we aim to extend the context sources, processor and reasoner components in various directions. The context source component will be extended to be able to use the ongoing work on the QoS context source proposed in [28]. Some other extensions include the context sources providing the monetary cost to use the network and energy requirements to use the available network interfaces. We are currently also investigating the use of various policies as proposed in [2], [4] and [6] to extend the Context Reasoner component. The future work also includes identifying the additional resource usage on the mobile device by the context-aware computing components introduced in this work.

\section{Acknowledgement}

This work is supported by Freeband Awareness project (under grant BSIK5902390) and Amigo project (IST-004182, partially funded by the European Commission). The authors thank to Richard Bults, Daniel Knoppel for integrating context-aware MSP components in the Awareness Integrated Health Demonstrator and providing valuable assistance for the experimental validation. The authors also like to thank to Kate Wac for providing valuable suggestions during this work. 


\section{References}

[1] Kaulgud, V. S. and S. A. Mondal., Exploiting multihoming for low latency handoff in heterogeneous networks, 8th International Conference on Telecommunications (ConTEL 2005), Zagreb, Croatia, 2005.

[2] Wakikawa, R., K. Uehara, et al., Multiple Network Interfaces Support by Policy-Based Routing on Mobile $I P v 6$, International Conference on Wireless Networks (ICWN 2002), Las Vegas USA. 2002,

[3] Mitsuya, K., K. Uehara, et al., The In-vehicle Router System to Support Network Mobility, Information Networking, Networking Technologies for Enhanced Internet Services International Conference (ICOIN 2003), Cheju Island, Korea. 2003.

[4] Ylitalo, J., T. Jokikyyny, et al., Dynamic Network Interface Selection in Multihomed Mobile Hosts, 36th Annual Hawaii International Conference on System Sciences, Island of Hawaii, USA, 2003.

[5] Montavont, N., T. Noel, and Ernst T., Multi-homing in Nested Mobile Networking, in Applications and the Internet Workshops co-located with SAINT 2004, Tokyo, Japan, 2004.

[6] Huang, C., Tsai, C., and Su., P., A Hybrid Approach For Ipv6 Multi-homing Providing F ault Tolerance And Load Balancing Capabilities, Second IASTED International Conference on Communication and Computer Networks, Cambridge, MA, USA, 2004.

[7] van Halteren A. T. and Pawar P., Mobile Service Platform: A Middleware for Nomadic Mobile Service Provisioning, 2nd IEEE International Conference On Wireless and Mobile Computing, Networking and Communications, Montreal, Canada, June 2006.

[8] Pratistha, D., Nicoloudis, N., Cuce, S., AMicro-Services Framework on Mobile Devices, International Conference on Web Services, Nevada, USA, 2003.

[9] Srirama, S. N., M. Jarke, et al., Mobile Web Service Provisioning, International Conference on Internet and Web Applications and Services (ICIW'06), Guadeloupe, French Caribbean, 2006.

[10] Alonso, A., Detailed Description of Trial Scenarios, D1.3, MobiHealth project (http://www.mobihealth.org), October 2002.

[11] Henricksen, K., J. Indulska, et al., Middleware for Distributed Context-Aware Systems, On the Move to Meaningful Internet Systems 2005, Agia Napa, Cyprus, 2005.

[12] Bults, R., Wac, K., van Halteren, A. T., Nicola, V. and Konstantas, D., Goodput Analysis of $3 G$ wireless networks supporting $m$-health services, proceedings of ConTEL 2005 - 8th International Conference on Telecommunications, Zagreb, Croatia. 15-17 June 2005.

[13] Kemerlis, V.P., et al. Throughput Unfairness in TCP over WiF $i$, Third Annual Conference on Wireless Ondemand Network Systems and Services (WONS 2006). Les Ménuires, France, 2006.

[14] Peddemors, I. Niemegeers, and Eertink, H., An Extensible Network Resource Abstraction for
Applications on Mobile Hosts, In Proceedings of the Second International Conference on Communication System Software and Middleware (COMSWARE'07), Jan. 2007.

[15] Sun Microsystems, The JINI Architecture Specification, http:/www.sun.com/software/JINI/specs/ JINI1_2.pdf, December 2001

[16] Sun Microsystems, JINI Technology Surrogate Architecture Specification, http://surrogate.JINI.org/sa.pdf, October 2003.

[17] Robert, C., KXML: A great find for xml parsing in J2ME, http://www.devx.com/xml/Article/11773/0/. 2003.

[18] JAKARTA Commons HTTPClient - The Apache Jakarta Project, http://jakarta.apache.org/commons/ httpclient/.

[19] Siau, K. and Shen, Z., Mobile Healthcare Informatics, Medical Informatics and the Internet in Medicine, 31(2): p. $89-99$, June 2006.

[20] Wegdam, M., AWARENESS: A project on Context AWARE mobile NEtworks and ServiceS, Mobile and Wireless Communication Summit, Dresden, Germany, 2005.

[21] Konstantas, D., Bults, R., Wac, K., Halteren, A. V., Final, Exploitation Ready MobiHealth BAN, D2.6, MobiHealth project (http://www.mobihealth.org), April 2004.

[22] Health Service 24 (eTEN-517352) project, http://www.healthservice24.com/cms/index.asp?healths ervice 24 .

[23] Widya, I., van Beijnum, B. J., Salden, A., QoC-based Optimization of End-to-End M-Health Data Delivery Services, 14th IEEE International Workshop on Quality of Service (IWQoS 2006), New Haven CT, June 2006.

[24] Marta Olivar Dimas et. al., Trial ready GPRS and UMTS networks (D 3.1), http:/www.mobihealth.org/ $\mathrm{html} /$ details/deliverables/pdf/new/MobiHealth_WP3_T ME_D3.1_v1_01.01.03.pdf, January 2003.

[25] Bandholz, M., Gefflaut, A., Riihijärvi, J., Wellens, M., and Mähönen, P., Unified Link-Layer API Enabling Wireless-Aware Applications, In Proceedings of the 17th International Symposium on Personal, Indoor and Mobile Radio Communications (PIMRC'06), September 2006.

[26] IETF Detecting Network Attachment (Active WG), $\mathrm{http}: /$ www.ietf.org/html.charters/dna-charter.html.

[27] IEEE 802.11 Working Group, http://www.ieee802.org/21/.

[28] Wac, K., van Halteren, A. T., Konstantas, D., QoSpredictions service: infrastructural support for proactive QoS- and context-aware mobile services, International Workshop on Context-Aware Mobile Systems (CAMS) collocated with On The Move 2006, Montpellier, France. 29 Oct - 3 Nov 2006. 